\title{
Rapid deployment of an emergency department-intensive care unit for the COVID-19 pandemic
}

\author{
Sean Hickey ${ }^{1}$, Kusum S. Mathews ${ }^{1-3}$, Jennifer Siller ${ }^{1}$, Judah Sueker ${ }^{1}$, \\ Mitali Thakore', Deepa Ravikumar', Ruben E Olmedo', Jolion McGreevy', \\ Roopa Kohli-Seth', Brendan Carr', Evan S. Leibner ${ }^{1,2}$

\begin{abstract}
'Department of Emergency Medicine, Icahn School of Medicine at Mount Sinai, New York, NY, USA ${ }^{2}$ Institute for Critical Care Medicine, Icahn School of Medicine at Mount Sinai, New York, NY, USA ${ }^{3}$ Division of Pulmonary, Critical Care, and Sleep Medicine, Department of Medicine, Icahn School of Medicine at Mount Sinai, New York, NY, USA
\end{abstract}

The coronavirus disease 2019 (COVID-19) pandemic mandated rapid, flexible solutions to meet the anticipated surge in both patient acuity and volume. This paper describes one institution's emergency department (ED) innovation at the center of the COVID-19 crisis, including the creation of a temporary ED-intensive care unit (ICU) and development of interdisciplinary COVID-19-specific care delivery models to care for critically ill patients. Mount Sinai Hospital, an urban quaternary academic medical center, had an existing five-bed resuscitation area insufficiently rescue due to its size and lack of negative pressure rooms. Within 1 week, the ED-based observation unit, which has four negative pressure rooms, was quickly converted into a COVID-19-specific unit, split between a 14-bed stepdown unit and a 13-bed ED-ICU unit. An increase in staffing for physicians, physician assistants, nurses, respiratory therapists, and medical technicians, as well as training in critical care protocols and procedures, was needed to ensure appropriate patient care. The transition of the ED to a COVID-19-specific unit with the inclusion of a temporary expanded ED-ICU at the beginning of the COVID-19 pandemic was a proactive solution to the growing challenges of surging patients, complexity, and extended boarding of critically ill patients in the ED. This pandemic underscores the importance of ED design innovation with flexible spacing, interdisciplinary collaborations on structure and services, and NP ventilation systems which will remain important moving forward.

Keywords COVID-19; Pandemics; Surge capacity; Emergency department; Intensive care units

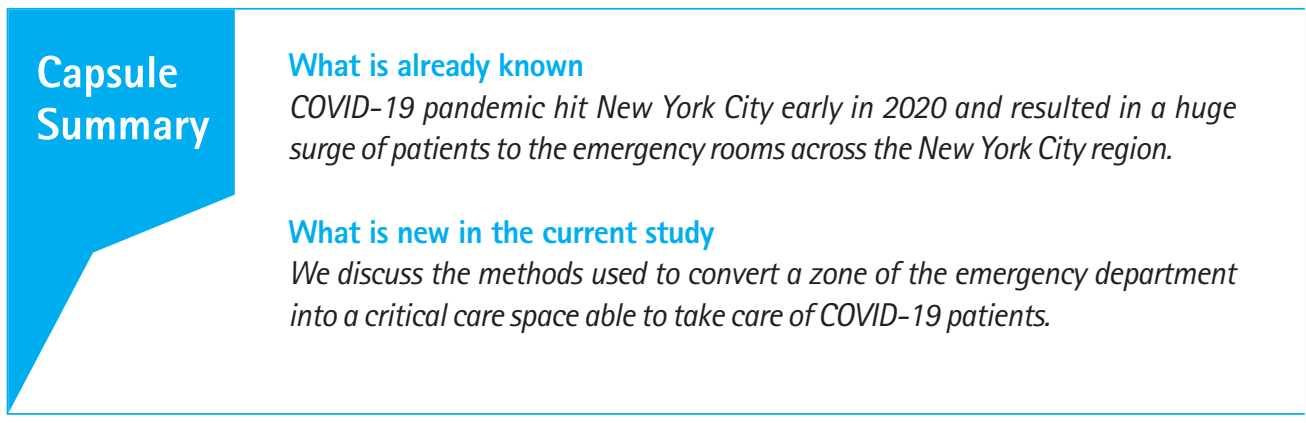

elSSN: 2383-4625

Received: 12 August 2020 Revised: 16 September 2020 Accepted: 3 October 2020

Correspondence to: Evan S Leibner Institute for Critical Care Medicine, Icahn School of Medicine at Mount Sinai, 1468 Madison Avenue, New York, NY 10029, USA

E-mail: evan.leibner@mountsinai.org ORCID

https://orcid.org/0000-0002-0490-1389

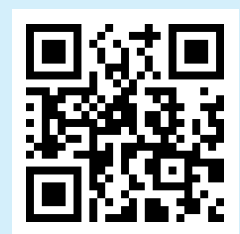

How to cite this article: Hickey S, Mathews KS, Siller J, Sueker J, Thakore M, Ravikumar D, Olmedo RE, McGreevy J, Kohli-Seth R, Carr B, Leibner ES. Rapid deployment of an emergency department-intensive care unit for the COVID-19 pandemic. Clin Exp Emerg Med 2020;7(4):319-325. https://doi. org/10.15441/ceem.20.102

This is an Open Access article distributed under the terms of the Creative Commons Attribution Non-Commercial License (https:// creativecommons.org/licenses/by-nc/4.0/). 


\section{INTRODUCTION}

Coronavirus disease 2019 (COVID-19), caused by the severe acute respiratory syndrome coronavirus 2 virus, has rapidly become a worldwide pandemic with over 44,000 COVID-19 confirmed cases and greater than 25,000 deaths in New York State as of September 10, 2020.'

The Mount Sinai Hospital (MSH) emergency department (ED) diagnosed the first New York State case of COVID-19 on March 1, 2020 in New York State. By April 1, the ED was admitting an average of 33 patients with COVID-19 to the hospital per day and discharging many more without confirmatory testing, in line with guidelines at that time. ${ }^{2,3}$ The physical layout of the ED was poorly suited to minimize the transmission risks of the COVID-19 virus with even more severely limited space for the high volume of critically ill patients. Thus, the pandemic forced a rapid adaptation and innovation of the ED physical space and workflows. ${ }^{4,5}$ This study details the ED's rapid structural changes, staffing transition, and physical updates for critically ill patients to offer lessons learned.

\section{SETTING}

The MSH ED is located on the upper east side of Manhattan, in New York City. It is an 1,139-bed quaternary care academic hospital with 104 intensive care unit (ICU) beds, typically operating at an average $>90 \%$ capacity. Over the past two decades, patient volumes have outgrown the 18,000-square foot department. The pre-pandemic layout of the department is detailed in Fig. 1. The adult MSH ED was divided into four main areas: one section for low acuity ambulatory patients, two sections for midacuity non-ambulatory patients, and a resuscitation area for high acuity patients (Fig. 1A). Adjacent to the adult ED was the pediatric ED and an observation unit. Across all ED sections, only ten rooms had respiratory isolation capabilities (i.e., negative pressure [NP] for airborne precautions). Due to high volume at baseline, physical space between critically ill patients was minimal throughout the department. During the 2 months between March and April 2019 the ED had 13,512 visits where during the COVID-19 pandemic that same period had 11,417 visits. The limitations of the ED size and layout were amplified during the pandemic.

\section{INITIAL CHALLENGES}

While the ED had a surge capacity plan prior to COVID-19, these emergency preparations were inadequate to meet the scale of the COVID-19 pandemic in New York City. The existing plans did not entail services, resources, and safeguards for such a large surge of critically ill patients, the high need for respiratory isolation, or the prolonged duration of disease activity. The principal challenge posed by COVID-19 was its transmissibility coupled with its unknown lethality and a lack of baseline population immunity. The initial goal for ED management was to accommodate every possible and confirmed COVID-19 patient (or family) in a NP room from arrival to discharge or admission. ${ }^{6-8}$ However, increased daily ED volume of suspected or confirmed COVID-19 cases-a rise of $86 \%$ by week 2 of the pandemic-led to a shortage of isolation rooms. This was further exacerbated by the increasing frequency of boarding by admitted ED patients awaiting inpatient bed availability in ward isolation rooms and units. Providers were forced to perform aerosolizing procedures either in bays open to the entire $E D$ or in a general isolation room without adequate ventilation, with limited options to transfer patients in and out of NP rooms due to the complexity of moving such unstable patients in a timely fashion. Furthermore, the shortage of NP rooms severely encumbered the initiation and utilization of non-invasive ventilation (NIV) (e.g., high-flow nasal cannula [HFNC] and non-invasive positive pressure ventilation [NIPPV]).

An additional challenge was ED crowding and boarding of inpatients, especially those on invasive mechanical ventilation. Prolonged boarding of ICU patients in the ED has been repeatedly associated with worse patient outcomes in prior studies. ${ }^{9-13} \mathrm{Un}$ surprising, daily arrivals of critically ill patients with COVID-19 outpaced the hospital's expansion of existing inpatient ICUs and construction of new ICUs. Compounding this situation, hospital cohorting required patients to wait for the in-house severe acute respiratory syndrome coronavirus two polymerase chain reaction assay results before unit and inpatient team assignments. Further, the critical care physicians who previously co-managed boarding ICU patients were deployed to care for patients in the expanded inpatient ICUs. ${ }^{14}$ As a result, the ED needed a significantly modified or expanded critical care space, change in the critical care staffing approach, and redesigned services to accommodate the ongoing influx and complex care of critically ill patients. ${ }^{15-18}$

\section{EVOLUTION OF THE SPACE}

The observation unit, adjacent to the ED, was an ideal place for critical care expansion due to existing access to the ambulance bay, four large NP rooms, and present infrastructure for critical care bay support. Supported by both ED and hospital leadership, steps began to convert this space into a temporary ED-based ICU. ${ }^{15,16,19}$ An ovenview of physical space changes is found in Table 1.

Thirteen critical care bays and 14 step-down beds were constructed in partnership from hospital engineering, biomedical sup- 


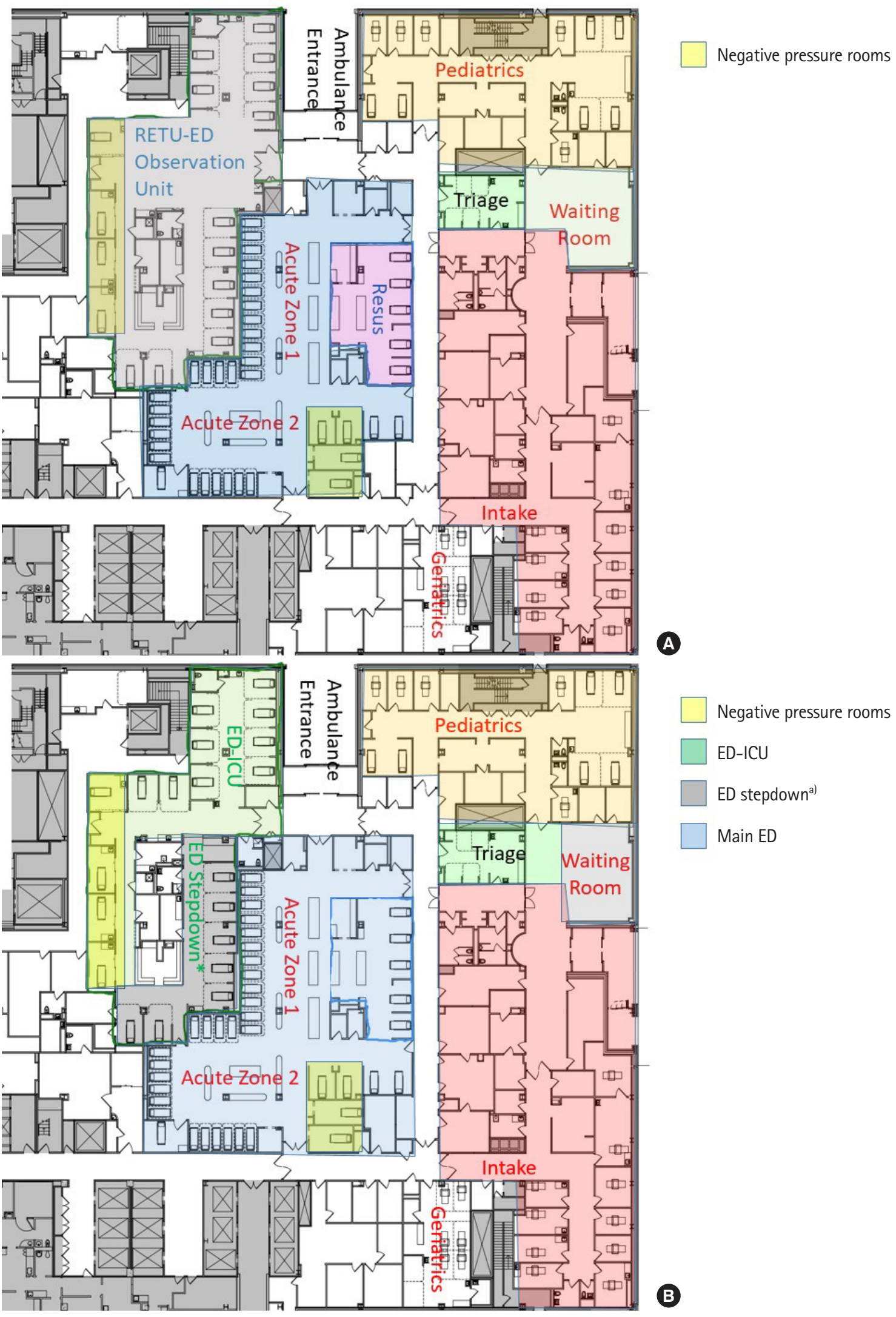

Fig. 1. (A) Pre-coronavirus disease 2019 (COVID-19) emergency department (ED) lay and (B) COVID-19 ED lay. ICU, intensive care unit. ${ }^{\text {a) }}$ Each bay in the stepdown zone represents two stepdown beds. 
plies, respiratory therapy, and supply management. The department of pharmacy, led by ED pharmacists, restocked the medications available in the new ED-ICU zone to include rapid sequence intubation agents, vasopressors, inotropes, paralytics, and sedatives.

Infection precautions for COVID-19 positive patients throughout the hospital were a combination of 'contact isolation' requiring a gown and gloves and 'special droplet' precautions requiring a surgical mask and face covering, plus an N-95 mask for aerosolizing procedures. N-95 masks and face shields were worn continuously in this zone to conserve personal protective equipment. Intubations required full personal protective equipment and to be done in a NP room, discarding all personal protective equipment except for the $\mathrm{N}-95$ mask after the procedure.

\section{STAFFING EXPANSION}

The severity of illness associated with COVID-19, nursing resources needed at the bedside during intubation or initiation of NIPPV, as well as requirements of minute-to-minute provision of ICU and stepdown level of care were all factors taken into consideration when developing the staffing model for the ED-ICU. An overview of staffing changes is found in Table 2 .

Table 1. ED critical care space

\begin{tabular}{lll}
\hline Space & \multicolumn{1}{c}{ Pre-COVID-19 } & COVID-19 pandemic \\
\hline ED critical care beds & 5 High acuity & 13 ICU \\
& 0 Stepdown & 14 Stepdown \\
Negative pressure rooms & 4 In observation unit & 4 In ED-ICU \\
& 4 In main ED & 4 In main ED \\
ED beds (total) & 76 & 102 \\
Hospital beds & 1,139 & 1,453 \\
Inpatient ICU beds & 98 & 235 \\
\hline
\end{tabular}

ED, emergency department; COVID-19, coronavirus disease 2019; ICU, intensive care unit.

\section{Nurse and ED technician staffing}

Nursing leadership determined that five nurses $24 / 7$ for the 27bed zone, with an additional three nurses 12 hours per day with staggered shifts during peak day hours was needed to staff the new ED-ICU. After direct feedback from frontline providers, nursing assignments were designated to certain rooms instead of the entire unit. New patients in need of immediate airway intervention received a dedicated nurse. Once intubated or on stable HFNC/ NIPPV settings, patients were handed off to a nurse covering multiple stable, critically ill patients. To help manage throughput, a sixth full-time registered nurse (RN), designated as Lead RN, was added at the end of the first week to the ED-ICU. Three full-time ED technicians provided ancillary support to the area.

Depending on critical care patient volume, the ED nursing director made real-time deployment decisions and shifted staff including RNs and technicians to the busiest areas in the department. Additional experienced nurses were deployed to the ED from closed procedural areas to support staffing the other areas of the ED while the primary ED RN staff were flexed to the ED-ICU.

\section{Provider staffing}

Given the anticipated volume and acuity, the new COVID-19 critical care section was staffed with expanded physician and advanced practice practitioner (APP) coverage. In contrast to the original model with only 11 hours a day of attending physician support, the new ED-ICU had full-time support by a dedicated emergency medicine attending and two trainees or APPs (either PGY-2 or above emergency medicine residents or senior physican assistants) 24/7. The new ED stepdown was staffed with a dedicated attending 16 hours per day and two EM residents or physican assistants. This staffing enabled two full resuscitations to occur simultaneously by two different ED attending's when needed.

MSH ED has six emergency physicians that are dual boarded in both emergency medicine and critical care medicine. While these

Table 2. ED-ICU provider staffing model

\begin{tabular}{lll}
\hline & \multicolumn{1}{c}{ Pre-COVID-19 } & \multicolumn{1}{c}{ COVID-19 pandemic } \\
\hline $\begin{array}{l}\text { Attending physicians } \\
\text { Trainees and advanced practice practitioners }\end{array}$ & $\begin{array}{l}\text { Dedicated physician only during peak hours } \\
1 \text { Upper year resident (PGY-2 or above) or senior APP }\end{array}$ & $\begin{array}{l}\text { Continuous coverage } \\
\text { Teams of } 2 \text { upper-year trainees or senior APPs for } \\
\text { ED-ICU and ED stepdown unit }\end{array}$ \\
Nurses & $3+$ Nurses at all times & $5+$ Nurses at all times \\
& $6+$ Nurses during peak hours. & $\begin{array}{l}\text { 8+ Nurses during peak hours } \\
1 \text { Senior nurse to manage flow }\end{array}$ \\
Respiratory therapists & 1 & 2 Plus CRNAs when available \\
ED technicians & 1 & 3
\end{tabular}

Changes in staffing models for management of critically ill ED patients from the pre-COVID-19 to the height of the pandemic.

ED, emergency department; ICU, intensive care unit; COVID-19, coronavirus disease 2019; PGY, post graduate year; APP, advance practice provider; CRNA, certified registered nurse anesthetist. 
physicians preferentially staffed the ED-ICU, inpatient ICU staffing demands led to the majority of ED-ICU shifts being covered by non-intensivist faculty. The ED intensivists worked to help develop novel protocols and adapt existing inpatient ICU for the critically ill COVID-19 patients in the pre-ICU setting, with implementation at all the EDs in the Mount Sinai Health System. ${ }^{20}$

\section{Respiratory therapy}

Prior to March, the ED had one dedicated respiratory therapist (RT) per shift. However, the volume of procedures and intubated patients required two full-time RTs with a third added for 12 hours a day during peak hours to help support the new demand. Several solutions were implemented to help support patient care for respiratory management, including ED physician training on a new ventilator and non invastive positive-pressure ventilaltion (NIPPV) machines setup and operation, hospital leadership allowance for a physician and RN to transport patients on portable ventilators and NIPPV without respitortory therapist (RT) assistance, and Operating Room CRNAs (certified registered nurse anesthetists) deployment to the ED to help with intubations and ventilator management.

\section{ELASTICITY OF SERVICES AND WORKFLOW}

\section{Triage}

In the beginning of the pandemic, it was posited that a subset of critically ill patients would have a low-enough pretest probability of COVID-19 that they should be separated from the high-likelihood COVID-19 patients. ${ }^{21}$ However, this split triage model was abandoned after a three-day trial due to the rapid drop-off in non-COVID-19 patients, ${ }^{22}$ and the high proportion of patients without respiratory complaints who nonetheless had COVID-19. All Emergency Severity Index 1 patients, and the majority of Emergency Severity Index 2 patients were triaged directly to the EDICU. Patients in need of rapid respiratory intervention (intubation or NIV initiation) were triaged directly into one of the four NP rooms. The remainder were triaged to the ED-ICU or stepdown

Table 3. Critically ill patient volume in the ED (March 1, 2020 to April $30,2020)$

\begin{tabular}{lrrrrrr}
\hline ED location & $\begin{array}{c}\text { Num- } \\
\text { ber }\end{array}$ & $\begin{array}{c}19+ \\
\text { COVID- }\end{array}$ & Male & $\begin{array}{c}\text { Admit- Expired Expired as of Sep- } \\
\text { ted }\end{array}$ & $\begin{array}{c}\text { in ED } \\
\text { tember 15, 2020 }\end{array}$ \\
\hline Peds & 1,575 & 31 & 791 & 181 & 0 & 1 \\
Stepdown & 468 & 200 & 263 & 313 & 2 & 81 \\
ED-ICU & 796 & 270 & 446 & 644 & 30 & 247 \\
Main (acute 1/2) & 4,509 & 965 & 2,251 & 1,961 & 3 & 280 \\
\hline
\end{tabular}

ED, emergency department; COVID-19, coronavirus disease 2019; ICU, intensive care unit. depending on their perceived stability. The ED-ICU and stepdown saw a large number of critical patients during the COVID-19 pandemic (Table 3).

\section{Respiratory resuscitation workflow}

During the first 5 weeks of the pandemic (March 22, 2020 to April 26, 2020), 878 patients were seen in this new space, with the number of intubated or NIV patients well exceeding the NP room capacity. Use of the four NP rooms was reserved for intubations and NIV use. In this time period, 127 patients required intubation in the $E D$, almost five times the number of patients intubated in 2019 (27). Once intubated, the patients were rapidly moved to one of the nine open ED-ICU bays where further care was provided. To further conserve limited NP space, intubated patients were deemed safe for regular isolation due to the ventilator's closed circuit with an attached viral filter. Similarly, patients on stable NIPPV or HFNC settings were moved to the four NP rooms in the other ED zones to save ED-ICU NP rooms for possible intubations.

Continuously moving patients out of the NP rooms to maintain their immediate availability for new arrivals was a great logistical challenge. Collaboration with environmental services was crucial to increase staffing to enable the cleaning and rapid turnover of the NP rooms which allowed for safe intubations. A nurse was assigned to solely manage the NP rooms to ensure that these critically ill patients received dedicated attention. This designated RN, the RTs and providers were able to continually assess for respiratory deterioration. Importantly, this nurse and the RT gained critical experience in rapidly moving recently intubated patients out of the NP rooms and providing nurse-to-nurse ICU-level handoffs in the ED-ICU and to the inpatient teams. The group that executed this transition included doctors, nurses, APPs, operational management staff and the ED performance improvement specialist. This group met daily, at a minimum, to ensure that we did not leave critical components out of the transition.

\section{Radiography}

All patients with suspected COVID-19 and any respiratory symptoms received a portable, anteroposterior chest radiograph. This was done to maintain the ED X-ray zone for non-respiratory plain films and reduce the deep cleaning needed between patients. Computerized tomography scans required coordination with the computerized tomography suite to maintain isolation precautions and protect the radiology staff.

\section{Palliative care}

Recognizing the need for expanded services with limited patient contact, the palliative care department initiated a 24/7 hotline 
for palliative care consults for all six Mount Sinai Health System EDs and deployed an in-person attending (often accompanied by a fellow or resident) to the MSH ED 12 hours per day. They functioned as core members of the team, continuously rounding and consulting on the majority of new patients as well as those with rapidly worsening clinical statuses. ${ }^{23}$ They assisted not only in helping to cognitively offload the stretched ED providers, at a time when patient's family could not be at the bedside due to infection risks, but also in helping providers feel that despite the crisis they were continuing to provide care consistent with patient/family wishes. ${ }^{24}$

\section{Admission flow and boarding time}

All hospital inpatient beds, including ICU beds, were rapidly divided into COVID-19 positive or negative units. The median turnaround time for this test was 7 hours, with a 95th percentile of 12 hours over the period in question. Additionally, whether due to collection technique or viral aggregation away from the upper airway, patients with negative COVID-19 swabs but with high suspicion of COVID-19 infection required repeated swabbing, thus extending boarding times. As such, no admitted patient could receive an inpatient bed assignment until a nasopharyngeal polymerase chain reaction resulted. Critically ill patients were similarly affected, as patients' infection status had to be confirmed before assignment to either a COVID-19 specialized ICU or a nonisolation ICU, increasing numbers of ICU patients boarded in the ED for longer than average times, requiring ED-specific innovation to meet the needs of the patients.

\section{DISCUSSION}

The MSH ED rapidly adapted the delivery of critical care to meet the challenges of the COVID-19 pandemic. ${ }^{20}$ An ED-ICU was rapidly built by converting the prior obsenvation area (a 27-bed zone). ${ }^{15,16,19,25}$ A redesign of a new space in a large US academic hospital often requires months, if not years, of planning and negotiations with the varying hospital interests involved. With the pressure of high COVID-19 demand, this conversion was executed in approximately 1 week, from initial decision to full activation. Moreover, the unit functioned during the peak of the New York City COVID-19 epidemic largely as envisioned and required surprisingly few spaces or workflow modifications mid-course. Its success was due to the hard work of the leadership team and front-line providers and the collaboration across the institution.

Strong intradepartmental and interdepartmental relationships were critical to the implementation of the ED-ICU unit. Success required the rapid cooperation of leadership from departments across the hospital. ED medical, nursing, and operations leadership had weekly meetings with all these departments. The transition to daily or twice-daily huddles required little change. With all these departments rapidly assisting to modify their own workflows and/or staffing, the new space achieved more optimal function, effectiveness, and safety for patients and staff with caregivers supported and operating in a more familiar environment.

There were and remain many challenges. Despite the relative strengths of the new space relative to the main ED, it is worth noting that not every COVID-19 patient was able to be treated behind a closed door, let alone in a NP room. Efforts to convert the whole zone to NP ventilation systems were deemed impossible given engineering constraints. As the process of redesigning the main ED begins-a process initiated before the pandemicthese lessons from the COVID-19 ED-ICU will better prepare the department for the future.

The COVID-19 pandemic was a strain on the healthcare system of New York City as a whole. The Mount Sinai Health System rapidly adapted to meet the needs of the patients. The ED rapidly designed and created an ED-ICU to help optimize critical care for the sickest patients presenting to the ED. The space was adapted to help facilitate safe, high-quality care for many critically ill patients, as well as providing continued ICU level care as these patients waited for ICU beds.

\section{CONFLICT OF INTEREST}

No potential conflict of interest relevant to this article was reported.

\section{ACKNOWLEDGMENTS}

We would like to thank Dr Jagoda for all his support, mentorship, and leadership on this manuscript. We would also like to thank Hospital Throughput, Engineering, Environmental Services, Internal Medicine, The Institute for Critical Care Medicine, Patient Transport, Radiology, Supply Chain Management, Respiratory Therapy, Security, Pharmacy, Laboratory, Office of the Chief Nursing Officer, Office of the Hospital President and CMO, Nutrition Services, and likely others who helped built and make the ED-ICU so successful.

\section{REFERENCES}

1. New York State Department of Health. New York State Department of Health COVID-19 tracker [Internet]. Albany, NY: New York State Department of Health; 2020 [cited 2020 Sep 
10]. Available from: https://covid19tracker.health.ny.gov/views/ NYS-COVID19-Tracker/NYSDOHCOVID-19Tracker-Map?\%3Ae mbed $=$ yesct\%3Atoolbar $=$ noct\%3Atabs $=$ n.

2. Tolia VM, Chan TC, Castillo EM. Preliminary results of initial testing for Coronavirus (COVID-19) in the emergency department. West J Emerg Med 2020;21:503-6.

3. Centers for Disease Control and Prevention. Overview of Testing for SARS-CoV-2 (COVID-19) [Internet]. Washington, DC: US Department of Health and Human Services; 2020 [cited 2020 May 21]. Available from: https://www.cdc.gov/coronavirus/2019-ncov/hcp/clinical-criteria.html.

4. Cao Y, Li Q, Chen J, et al. Hospital emergency management plan during the COVID-19 epidemic. Acad Emerg Med 2020; 27:309-11.

5. Paganini M, Conti A, Weinstein E, Della Corte F, Ragazzoni L. Translating COVID-19 pandemic surge theory to practice in the emergency department: how to expand structure. Disaster Med Public Health Prep 2020:1-10.

6. Alhazzani W, Moller MH, Arabi YM, et al. Surviving sepsis campaign: guidelines on the management of critically III adults with coronavirus disease 2019 (COVID-19). Crit Care Med 2020;48:e440-69.

7. Raffensperger JF, Brauner MK, Briggs RJ. Planning hospital needs for ventilators and respiratory therapists in the COVID-19 crisis. Santa Monica, CA: RAND Corporation; 2020.

8. Hartnett KP, Kite-Powell A, DeVies J, et al. Impact of the COVID-19 pandemic on emergency department visits: United States, January 1, 2019-May 30, 2020. MMWR Morb Mortal Wkly Rep 2020;69:699-704.

9. Anesi GL, Liu VX, Gabler NB, et al. Associations of intensive care unit capacity strain with disposition and outcomes of patients with sepsis presenting to the emergency department. Ann Am Thorac Soc 2018;15:1328-35.

10. Mathews KS, Durst MS, Vargas-Torres $C$, Olson AD, Mazumdar M, Richardson LD. Effect of emergency department and ICU occupancy on admission decisions and outcomes for critically ill patients. Crit Care Med 2018;46:720-7.

11. Chalfin DB, Trzeciak S, Likourezos A, Baumann BM, Dellinger RP; DELAY-ED study group. Impact of delayed transfer of critically ill patients from the emergency department to the intensive care unit. Crit Care Med 2007;35:1477-83.

12. Cardoso LT, Grion CM, Matsuo T, et al. Impact of delayed admission to intensive care units on mortality of critically ill patients: a cohort study. Crit Care 2011;15:R28.

13. Hung SC, Kung CT, Hung CW, et al. Determining delayed admission to intensive care unit for mechanically ventilated patients in the emergency department. Crit Care 2014;18:485.
14. Vincent JL. The continuum of critical care. Crit Care 2019; 23(Suppl 1):122.

15. Leibner $\mathrm{E}$, Spiegel $\mathrm{R}$, Hsu $\mathrm{CH}$, et al. Anatomy of resuscitative care unit: expanding the borders of traditional intensive care units. Emerg Med J 2019;36:364-8.

16. Gunnerson KJ, Bassin BS, Havey RA, et al. Association of an emergency department-based intensive care unit with survival and inpatient intensive care unit admissions. JAMA Netw Open 2019;2:e197584.

17. Gunnerson KJ. The emergency department's impact on inpatient critical care resources. Acad Emerg Med 2017;24:1283-5.

18. National Science and Technology Council. Towards epidemic prediction: federal efforts and opportunities in outbreak monitoring [Internet]. Washington, DC: National Science and Technology Council; 2016 [cited 2020 May 21]. Available from: https://obamawhitehouse.archives.gov/sites/default/files/microsites/ostp/NSTC/towards_epidemic_prediction-federal_efforts_and_opportunities.pdf.

19. Weingart SD, Sherwin RL, Emlet LL, Tawil I, Mayglothling J, Rittenberger JC. ED intensivists and ED intensive care units. Am J Emerg Med 2013;31:617-20.

20. Leibner ES, Stokes $S, A$ Ahad D, Legome E. Emergency department COVID management policies: one institution's experience and lessons learned. Emerg Med Pract 2020;22(5 Suppl):1.

21. Wee LE, Fua TP, Chua YY, et al. Containing COVID-19 in the emergency department: the role of improved case detection and segregation of suspect cases. Acad Emerg Med 2020;27: 379-87.

22. Wong LE, Hawkins JE, Langness $S$, Murrell KL, Iris $P$, Sammann A. Where are all the patients?: addressing Covid-19 fear to encourage sick patients to seek emergency care. NEJM Catal Innov Care Deliv 2020 May 14. https://doi.org/10.1056/CAT. 20.0193.

23. Ankuda CK, Woodrell CD, Meier DE, Morrison RS, Chai E. A beacon for dark times: palliative care support during the coronavirus pandemic. NEJM Catal Innov Care Deliv 2020 May 12. https://doi.org/10.1056/CAT.20.0204.

24. Santarone K, McKenney M, Elkbuli A. Preserving mental health and resilience in frontline healthcare workers during COVID-19. Am J Emerg Med 2020;38:1530-1.

25. Puy L, Lamy C, Canaple $S$, et al. Creation of an intensive care unit and organizational changes in an adult emergency department: impact on acute stroke management. Am J Emerg Med 2017;35:716-9. 